If the conjecture is valid, then there exists a counterexample showing that a certain reduced gradient algorithm for solving linearly constrained minimization problems does not exhibit linear convergence (M. Gaviano, B. Piazza, On the rate of convergence of a reduced gradient method, Boll. Unione Matematica Italiana, to appear).

\title{
On the Expected Value of Two Statistics
}

Problem 82-13, by K. A. RaO (State University of New York at Binghamton). If

$$
\begin{aligned}
& R_{2}=\max \left(Y_{1}, Y_{2}\right)-\min \left(Y_{1}, Y_{2}\right), \\
& R_{3}=\max \left(Y_{1}, Y_{2}, Y_{3}\right)-\min \left(Y_{1}, Y_{2}, Y_{3}\right),
\end{aligned}
$$

where $Y_{1}, Y_{2}$, and $Y_{3}$ are cyclically exchangeable random variables, prove that

$$
2 E\left(R_{3}\right)=3 E\left(R_{2}\right) .
$$

This problem generalizes a result of $\mathrm{H}$. E. Robbins for the i.i.d. case (see On the expected value of two statistics, Ann. Math. Statist., 15 (1944), pp. 321-323).

\section{A Conjecture on the Number of Real Zeros}

Problem 82-14*, by T. C. Y. LAU (University of Hong Kong)

It is conjectured that the function

$$
P_{m}(x)=e^{-x} \prod_{i=1}^{n}\left(1+b_{i} x\right)
$$

cannot have more than $m+2$ real zeros on the nonnegative real axis. Here, $P_{m}(x)$ is any polynomial of degree at most $m$ and $b_{i}, i=1,2, \cdots, n$ are $n$ positive real numbers. Prove or disprove.

The problem arose in trying to approximate exponential functions by rational polynomials with real poles. The special case $m=0$ has been solved.

\section{Flight in an Irrotational Wind Field. II}

\section{Problem 82-15, by M.S. KLAMKIN (University of Alberta).}

It is a known result (see Problem 61-4, SIAM Rev., 4 (1962), p. 155) that if an aircraft traverses a closed curve at a constant air speed with respect to the wind, the time taken is always less when there is no wind, than when there is any bounded irrotational wind field.

(i) Show more generally that if the wind field is $k \mathbf{W}$ (W bounded and irrotational and $k$ is a constant), then the time of traverse is a monotonic increasing function of $k$ $(k \geqq 0)$.

(ii) Let the aircraft be subject to the bounded irrotational wind field $\mathbf{W}_{i}, i=1,2$, and let $T_{i}$ denote the time of flight over the same closed path. If $\left|\mathbf{W}_{1}\right| \leqq\left|\mathbf{W}_{2}\right|$ at every point of the traverse, does it follow that $T_{1} \leqq T_{2}$ ?

\section{SOLUTIONS}

\section{Some Inequalities Involving Statistical Expressions}

Problem 81-10, by L. V. FosTER (University of Pittsburgh at Johnstown).

For a set of nonnegative numbers $u_{1}, u_{2}, \cdots, u_{n}$, let $u=\left(u_{1}+u_{2}+\cdots+u_{n}\right) / n$ 
be the arithmetic mean, let $\rho=\left(u_{1} u_{2} \cdots u_{n}\right)^{1 / n}$ be the geometric mean and let $s_{m}=\left[\sum_{j=1}^{n}\left(u_{j}-u\right)^{2} /(n \cdot(n-1))\right]^{1 / 2}$ be the sample standard deviation of the mean. It is well known that $u \geqq \rho$. Prove the stronger inequalities

$$
u^{n} \geqq\left[u+(n-1) s_{m}\right]\left(u-s_{m}\right)^{n-1} \geqq \rho^{n} \geqq\left[u-(n-1) s_{m}\right]\left(u+s_{m}\right)^{n-1} .
$$

These inequalities arose in studying an iterative method for finding zeros of polynomials.

Solution by J. M. Borwein (Carnegie-Mellon University), G. P. H. StYAn (McGill University), and $\mathrm{H}$. WoLKOWICZ (University of Alberta).

We may, without loss of generality, assume that the $u_{i}$ 's are ordered: $u_{1} \geqq \cdots \geqq u_{n} \geqq 0$. Then $u_{n} \leqq u-s_{m}$ from Samuelson's inequality [1, eq. (1.2)], and so $u-s_{m} \geqq 0$. The first inequality in (1), therefore, follows at once from the arithmetic mean/geometric mean inequality:

$$
u^{n}=\left\{\frac{1}{n}\left[u+(n-1) s_{m}+(n-1)\left(u-s_{m}\right)\right]\right\}^{n} \geqq\left[u+(n-1) s_{m}\right]\left(u-s_{m}\right)^{n-1},
$$

with equality if and only if $s_{m}=0$ or $u_{1}=\cdots=u_{n}$.

To prove the other inequalities in (1) we first note that

$$
u+(n-1) s_{m} \geqq u_{1} \geqq u+s_{m},
$$

cf. [1, eqs. (1.1) and (1.3)]. Equality on the left of (3) occurs if and only if

$$
u_{1}=u+(n-1) s_{m} \text { and } u_{2}=\cdots=u_{n}=u-s_{m},
$$

and on the right if and only if

$$
u_{1}=\cdots=u_{n-1}=u+s_{m} \text { and } u_{n}=u-(n-1) s_{m} .
$$

Let

$$
q^{2}=\sum u_{i}^{2}=n(n-1) s_{m}^{2}+n u^{2}>0
$$

and $u$ be fixed, and let

$$
\mathcal{U}_{n}=\left\{\left(u_{i}\right): u_{1} \geqq \cdots \geqq u_{n} \geqq 0 ; \sum u_{i}=n u, \sum u_{i}^{2}=q^{2}\right\} .
$$

Since $\mathcal{U}_{n}$ is compact and $q^{2}>0$, the maximum of $\rho^{n}$ is attained in $\mathcal{U}_{n}$, at $\hat{u}_{1} \geqq \cdots \geqq \hat{u}_{n}>0$, say.

Our proof of the second inequality in (1) will be by contradiction. Suppose that $\hat{u}_{1}$ is not at its maximum $u+(n-1) s_{m}$ and so $\hat{u}_{2}>\hat{u}_{n}$. Then, by (3) and (4), there exists a set of $n$ numbers: $v_{1}, v_{2}, \cdots, v_{n}$, with $v_{1} \geqq v_{2} \geqq v_{n}$ and such that $v_{1}>\hat{u}_{1}, v_{i}=\hat{u}_{i}(i \neq 1,2, n)$ and $v_{1}+v_{2}+v_{n}=\hat{u}_{1}+\hat{u}_{2}+\hat{u}_{n}$, and $v_{1}^{2}+v_{2}^{2}+v_{n}^{2}=\hat{u}_{1}^{2}+\hat{u}_{2}^{2}+\hat{u}_{n}^{2}$; a permutation of $v_{1}, v_{2}, \cdots, v_{n}$ lies in $\boldsymbol{u}_{n}$.

Holding $u_{3}, \cdots, u_{n-1}$ fixed, the partial derivative with respect to $u_{1}$ of

$$
\begin{aligned}
\rho^{n} & =u_{1} u_{2} \cdots u_{n} \\
& =1 / 2-u_{1}\left[\left(n u-u_{1}-u_{3}-\cdots-u_{n-1}\right)^{2}\right. \\
& \left.\quad\left(q^{2}-u_{1}^{2}-u_{3}^{2}-\cdots-u_{n-1}^{2}\right)\right] u_{3} \cdots u_{n-1}
\end{aligned}
$$

is

$$
\frac{\partial \rho^{n}}{\partial u_{1}}=\left(u_{1}-u_{2}\right)\left(u_{1}-u_{n}\right) u_{3} \cdots u_{n-1} \geqq 0,
$$

and so $\rho^{n}$ is nondecreasing in $u_{1}$. Similarly, it follows that 


$$
\frac{\partial \rho^{n}}{\partial u_{2}}=\left(u_{2}-u_{1}\right)\left(u_{2}-u_{n}\right) u_{3} \cdots u_{n-1} \leqq 0,
$$

and $\rho^{n}$ is nonincreasing in $u_{2}$. Thus $u_{2}$ is nonincreasing in $u_{1}$ and so

$$
v_{1}>\hat{u}_{1} \geqq \hat{u}_{2} \geqq v_{2} \text {. }
$$

Let $v_{1}=\hat{u}_{1}+\varepsilon$, with $\varepsilon>0$. Then

$$
\begin{aligned}
\hat{u}_{1} \hat{u}_{2} \hat{u}_{n} & =1 / 2\left(v_{1}-\varepsilon\right)\left[\left(v_{2}+v_{n}+\varepsilon\right)^{2}-\left\{v_{1}^{2}+v_{2}^{2}+v_{n}^{2}-\left(v_{1}-\varepsilon\right)^{2}\right\}\right] \\
& =-\varepsilon^{3}+\varepsilon^{2}\left(2 v_{1}-v_{2}-v_{n}\right)-\varepsilon\left(v_{1}-v_{2}\right)\left(v_{1}-v_{n}\right)+v_{1} v_{2} v_{n} \\
& <v_{1} v_{2} v_{n}
\end{aligned}
$$

for sufficiently small $\varepsilon>0$ since $v_{1}>v_{2}$ by (11). Hence the maximum of $\rho^{n}$ cannot be attained at $\hat{u}_{1} \geqq \cdots \geqq \hat{u}_{n}$ unless $\hat{u}_{1}$ equals the maximum of $u_{1}$ in $\mathcal{u}_{n}$. The middle inequality in (1) then follows from (4), which also characterizes equality.

The right inequality in (1) is trivial when $u \leqq(n-1) s_{m}$. So let

$$
u>(n-1) s_{m} .
$$

(For an interpretation of (13) see [2, p. 486].) Then by the inequality

$$
u_{n} \geqq u-(n-1) s_{m}
$$

$[1,(1.2)]$ it follows that $u_{n}>0$ and hence it follows from (12), reversing the order and roles of the $\hat{u}_{i}$ and $v_{i}$ (so that the $v_{i}$ identify the minimum of $\rho^{n}$ ), that the right inequality in (1) holds, with equality if and only if (5) holds.

Remarks. The nonnegative numbers $u_{i}$ can be considered to be the eigenvalues of a Hermitian nonnegative definite matrix A (cf. [2, p. 484]), or the roots of a polynomial $p(x)=x^{n}-a_{0} x^{n-1}+a_{1} x^{n-2}+\cdots+(-1)^{n} a_{n}$. In the case of a matrix $\mathbf{A}$, we have $\rho^{n}=\operatorname{det} \mathbf{A}$, while $u=\operatorname{tr} \mathbf{A} / n$, and $s_{m}^{2}=\left(\operatorname{tr} \mathbf{A}^{2}-n u^{2}\right) /[n(n-1)]$, where det denotes determinant and tr denotes trace. In the case of a polynomial, we have $\rho^{n}=a_{n}$, while $u=a_{0} / n$ and $s_{m}^{2}=\left[2 a_{1}-(n+1) a_{0}^{2}\right] /[n(n-1)]$. Foster's inequalities (1) provide information on the eigenvalues or roots. For example, if one of the inequalities among the coefficients of the polynomial fails, then the roots cannot all be nonnegative.

Moreover, the right inequality in (1) is essentially the same as [2, eq. (2.58), p. 485], which strengthens the comparison about two upper bounds for the condition number of a Hermitian positive definite matrix.

\section{REFERENCES}

[1] H. Wolkowicz and G. P. H. Styan, Reply: On extensions of Samuelson's inequality, Amer. Statist., 34 (1980), pp. 250-251.

[2] —_, Bounds for eigenvalues using traces, Linear Algebra Appl., 29 (1980), pp. 471-506.

Also solved by the proposer.

\section{Estimation of the Median Effective Dose in Bioassay}

Problem 81-12, by D. E. RAESIDE (University of Oklahoma, Health Sciences Center).

Consider a system subjected to a single stimulus intensity (dose level) $x$, and assume that the system responds in just one of two ways, "positively" or "negatively". Let the 